Revue des patrimoines

43 | 2021

Des écoles d'art académiques aux écoles d'art : des collections et des lieux, un patrimoine à valoriser

\title{
L'Accademia Albertina di Belle Arti de Turin
}

Ses origines, son histoire, ses collections

The Accademia Albertina di Belle Arti of Turin. Its origins, its history, its

collections

\section{Marco Carassi}

\section{(2) OpenEdition}

Journals

Édition électronique

URL : http://journals.openedition.org/insitu/29201

DOI : 10.4000/insitu.29201

ISSN : 1630-7305

Éditeur

Ministère de la Culture

Référence électronique

Marco Carassi, « L'Accademia Albertina di Belle Arti de Turin », In Situ [En ligne], 43 | 2021, document 13, mis en ligne le 12 janvier 2021, consulté le 11 février 2021. URL : http://journals.openedition.org/ insitu/29201; DOI : https://doi.org/10.4000/insitu.29201

Ce document a été généré automatiquement le 11 février 2021.

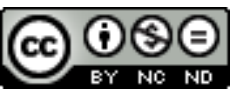

In Situ Revues des patrimoines est mis à disposition selon les termes de la licence Creative Commons Attribution - Pas d'Utilisation Commerciale - Pas de Modification 4.0 International. 


\title{
L'Accademia Albertina di Belle Arti de Turin
}

\author{
Ses origines, son histoire, ses collections \\ The Accademia Albertina di Belle Arti of Turin. Its origins, its history, its \\ collections
}

Marco Carassi

1 Le 29 août 1678, Marie Jeanne Baptiste de Savoie-Nemours, régente des États de la maison de Savoie après la mort du duc Charles-Emmanuel II, institutionnalise une ancienne corporation de métier en créant l'« Académie des peintres, des sculpteurs et des architectes" de Turin ${ }^{1}$. La première collection d'œuvres d'art destinée à l'enseignement est formée par les donations auxquelles sont tenus les académiciens, hommes et femmes. Sous Victor-Amédée II, en 1716, ses statuts, inspirés de ceux de l'Académie romaine de Saint Luc, sont approuvés².

2 En 1778, l'Académie est refondée, à l'initiative du comte Giuseppe Roberto Berthoud de Malines, très critique à propos de l'état auquel avait été réduite l'institution après un siècle d'existence: "Il ne manquoit que peu de chose à l'école de peinture: un maître et des élèves ${ }^{3}$. " La direction, confiée à cette occasion au peintre français de formation romaine Laurent Pécheux (1729-1821), se caractérise par des liens étroits avec les Académies de Rome, Parme et Paris. C'est à lui qu'on doit la formation d'une gypsothèque par l'achat, à Rome, de copies de statues anciennes, dont on connaît une liste de vingt-quatre plâtres, datée de 1811. Pécheux nomme Carlo Antonio Porporati responsable de l'école de gravure, Giuseppe Duprà de l'école de peinture, Ignazio et Filippo Collino de l'école de sculpture ${ }^{4}$.

3 Joseph-Jérôme Lefrançois de Lalande, dans son Voyage en Italie (1786), décrit l'école et son corps d'enseignants 5 . À l'époque napoléonienne, lorsque le Piémont est annexé à la France, l'Académie de Turin est intégrée au système universitaire impérial. En 1808, l'École spéciale des arts du dessin de Turin est rattachée à l'Académie de Paris ${ }^{6}$. Les cours sont donnés en ville, dans différents lieux, par des enseignants renommés tels le sculpteur Giacomo Spalla et l'architecte Ferdinando Bonsignore. La «petite 
bibliothèque » dont parle Pécheux pour l'instruction des élèves comprend la Bible, les œuvres d'Homère, Virgile et Ovide, nombre de traités d'histoire ancienne et moderne, des biographies d'artistes, des répertoires d'iconologie pour la peinture et la sculpture [fig. 1]. Donations de livres, gravures et lithographies se succèdent selon les tendances artistiques au fil de leur apparition. Pour une période limitée, un Musée didactique de sculptures et tableaux est établi au Palais impérial (ex-royal) de Turin, tandis qu'en ville, à l'occasion des passages de Napoléon, on organise des expositions d'« Objets d'art, manufactures et métiers ». Les expositions annuelles des œuvres des élèves de l'Académie ont lieu dans l'ancien couvent de Saint-François-de-Paule? ${ }^{7}$.

Figure 1

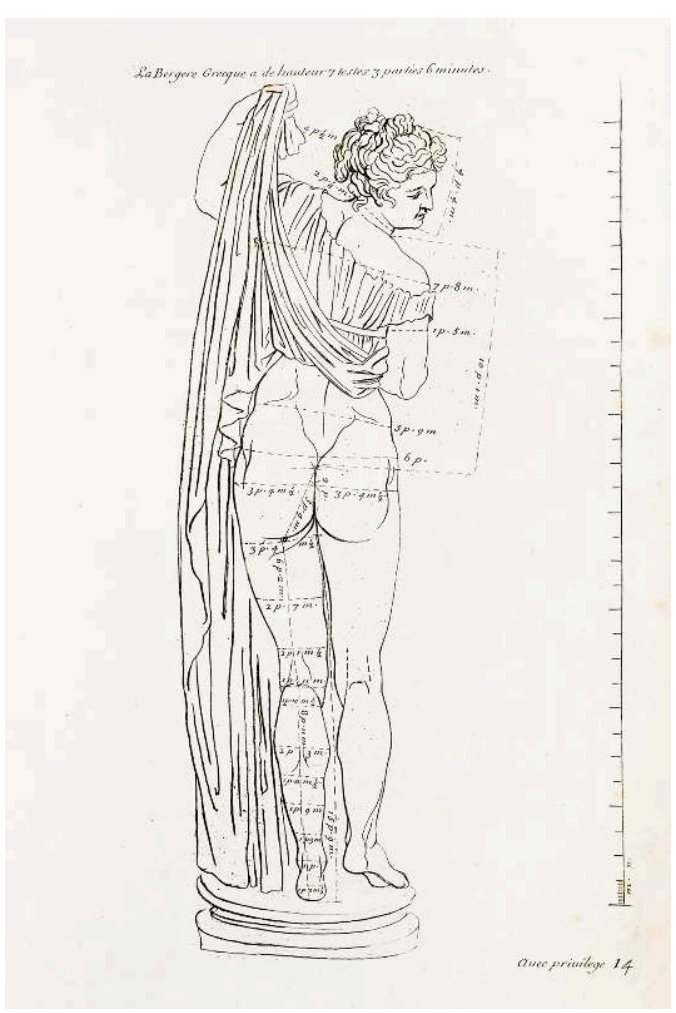

La Bergère grecque, ou Vénus callipyge, page extraite de AUDRAN Gérard, Les Proportions du corps humain mesurées sur les plus belles figures de l'Antiquité, Paris, Joubert, 1801 [1683], planche 14. L'image porte les mesures utiles aux sculpteurs.

En 1820, le roi de Sardaigne et duc de Savoie Charles-Félix remet en vigueur les règlements de 1778 et nomme secrétaire perpétuel de l'Académie le peintre niçois Giovanni Battista Biscarra, qui exerce une profonde influence jusqu'en 1851. En 1833, son successeur Charles-Albert installe définitivement l'Académie dans l'ancien couvent de Saint-François-de-Paule ${ }^{9}$ (où elle se trouve encore) [fig. 2] et lui fait don en 1837 de l'ouvrage de Winckelmann sur l'art du dessin chez les anciens ${ }^{10}$. 
Figure 2

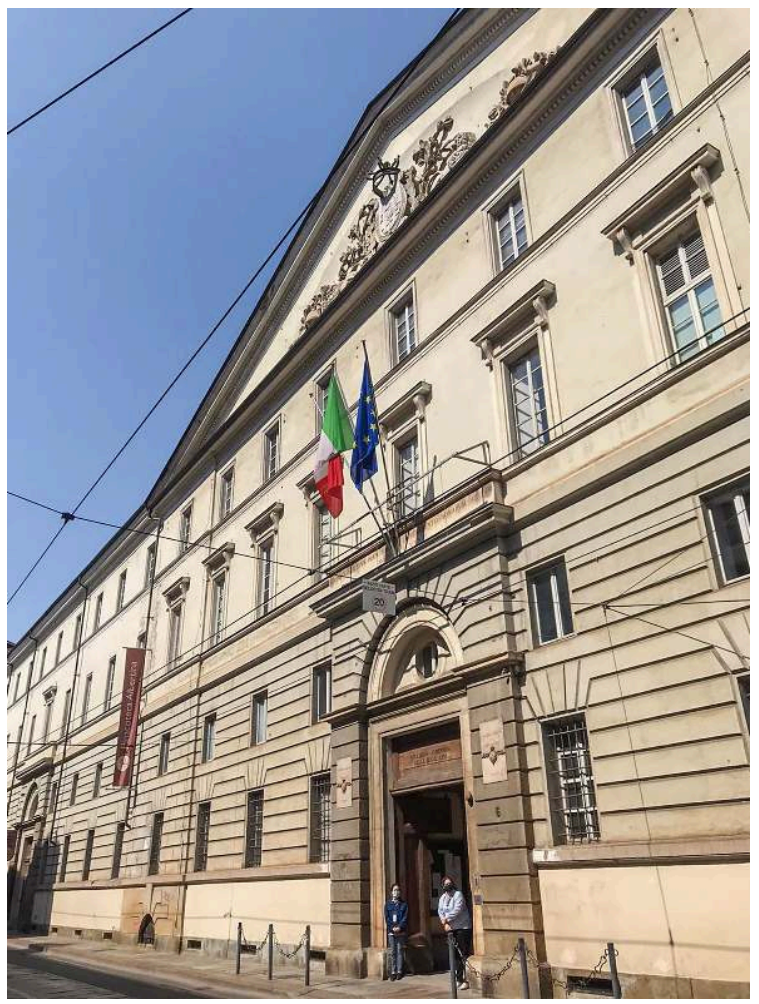

Accademia Albertina, façade principale.

(c) photo Fabio Amerio (Accademia Albertina di Belle Arti, Turin).

5 L'architecture du bâtiment, construit au XVII ${ }^{e}$ siècle, est adaptée à plusieurs reprises : à partir de 1822 par l'architecte Giuseppe Maria Talucchi (on lui doit la nouvelle façade monumentale et l'imposante "Rotonda » située dans la grande cour [fig. 3]); après 1834, par les architectes Carlo Bernardo Mosca et Ernesto Melano (entrée à colonnes, salle du roi Charles-Albert et escalier monumental [fig. 4, fig. 5]); après 1856, à la demande du sculpteur Vincenzo Vela, la salle-atelier est agrandie. Et surtout, on procède à de nouveaux travaux, indispensables, après les dégâts considérables occasionnés par les bombardements de $1944^{11}$. 
Figure 3

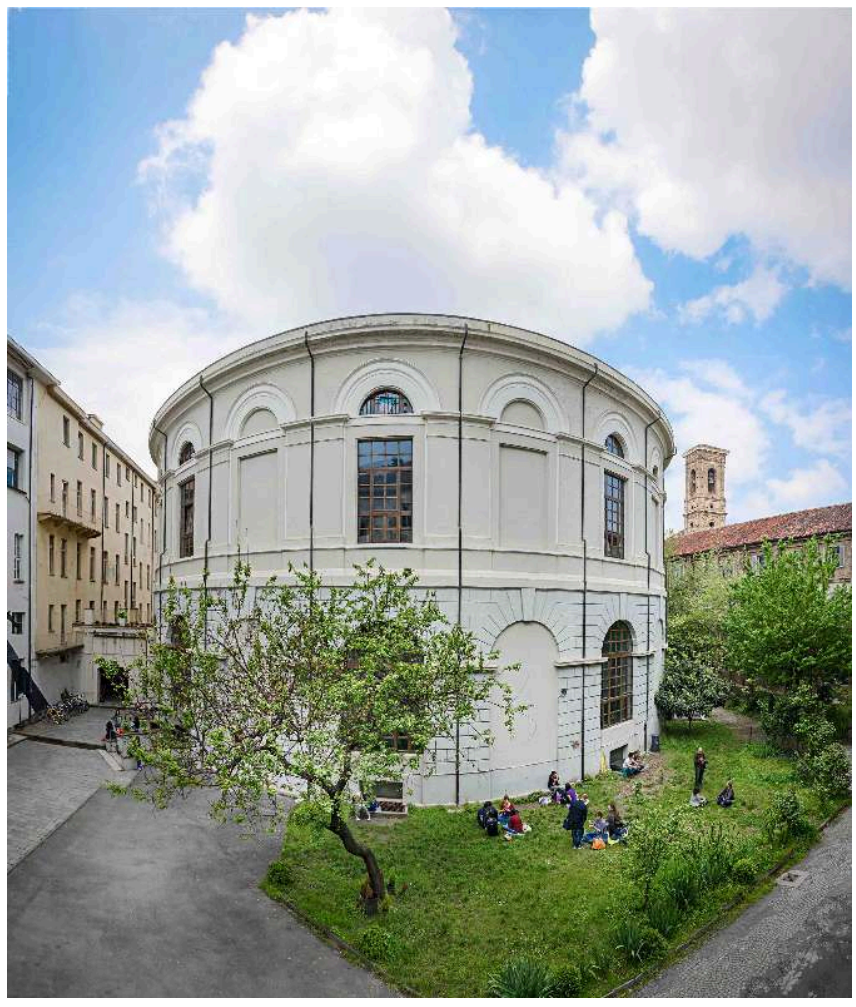

Accademia Albertina, la « Rotonda ».

(C) photo Fabio Amerio (Accademia Albertina di Belle Arti, Turin).

\section{Figure 4}

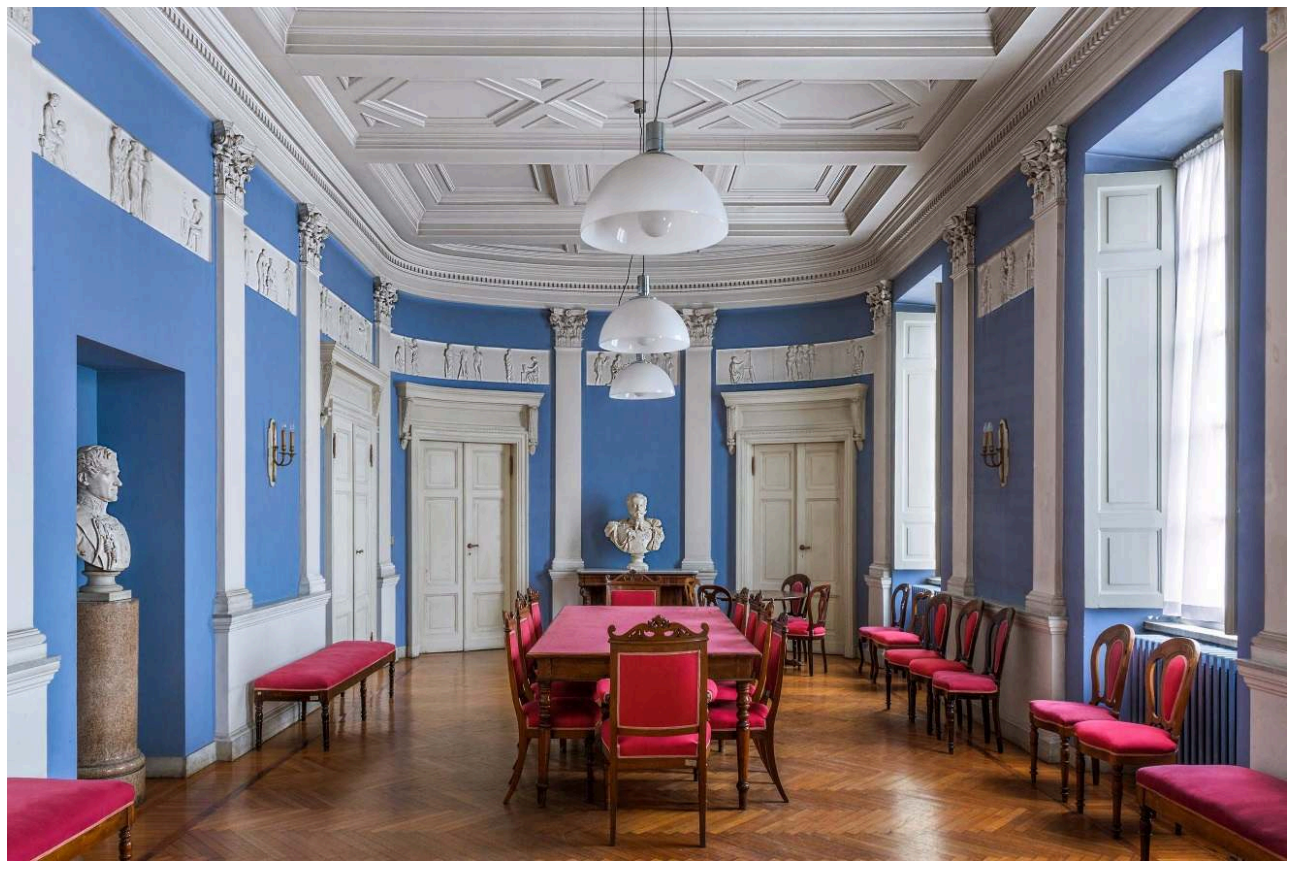

Accademia Albertina, salle avec buste du roi Charles-Albert de Savoie.

(C) photo Fabio Amerio (Accademia Albertina di Belle Arti, Turin). 
Figure 5

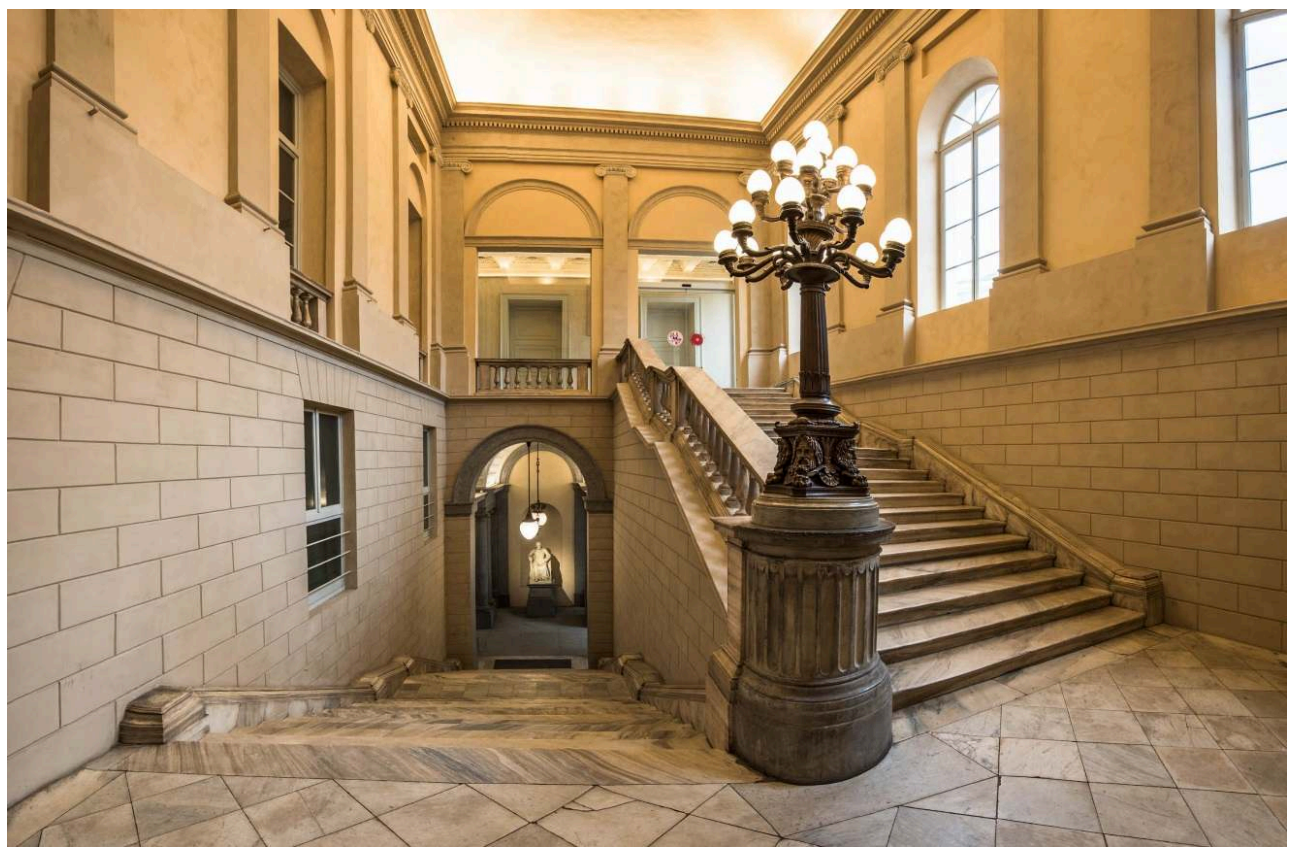

Accademia Albertina, escalier monumental.

(C) photo Fabio Amerio (Accademia Albertina di Belle Arti, Turin).

L'Académie est refondée en 1824 et rendue autonome de l'université. On admet, parmi les académiciens, les plus importants collectionneurs et commanditaires d'œuvres d'art. À l'époque du roi Charles-Albert de Savoie, la collection des plâtres de l'Académie, devenue "Albertina », placés dans les salles des écoles de sculpture, d'anatomie et de nu (dessin d'après modèle vivant), atteint les 850 œuvres (inventaires de 1839, 1846 et 1856) ${ }^{12}$, en grande partie détruites par les bombardements de la Deuxième Guerre mondiale. Dans les années 1830, l'enseignement de l'anatomie est confié à Francesco Bertinatti [fig. 6] dont les manuscrits rédigés entre 1837 et 1839 sont conservés dans le fonds ancien de la bibliothèque de l'Académie, [fig. 7] ainsi que les deux volumes publiés par l'éditeur Marietti en 1837 et les additions, avec 37 lithographies, publiées jusqu'en 1839, œuvres qui sont présentes en plusieurs exemplaires pour l'usage des élèves ${ }^{13}$. 
Figure 6

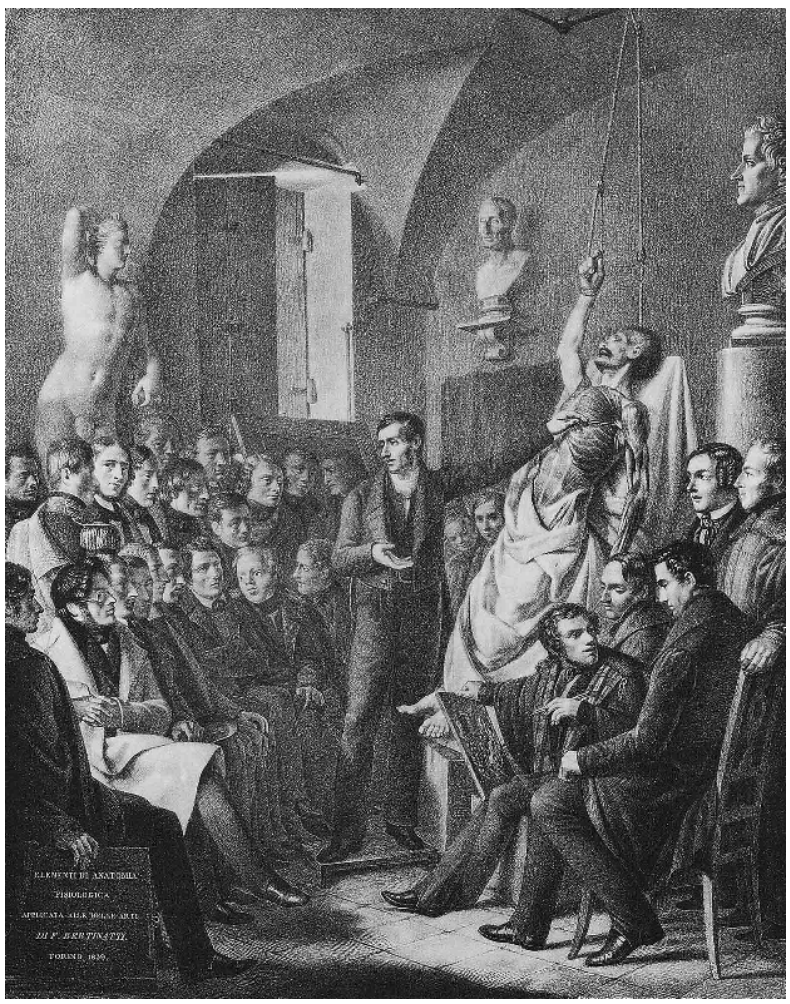

Leçon d'anatomie, Paolo Emilio Morgari, lithographie d'ouverture extraite de l'ouvrage de BERTINATTI Francesco, Elementi di anatomia fisiologica applicata alle Belle Arti figurative, Turin, Pietro Marietti, 1837-1839.

Figure 7

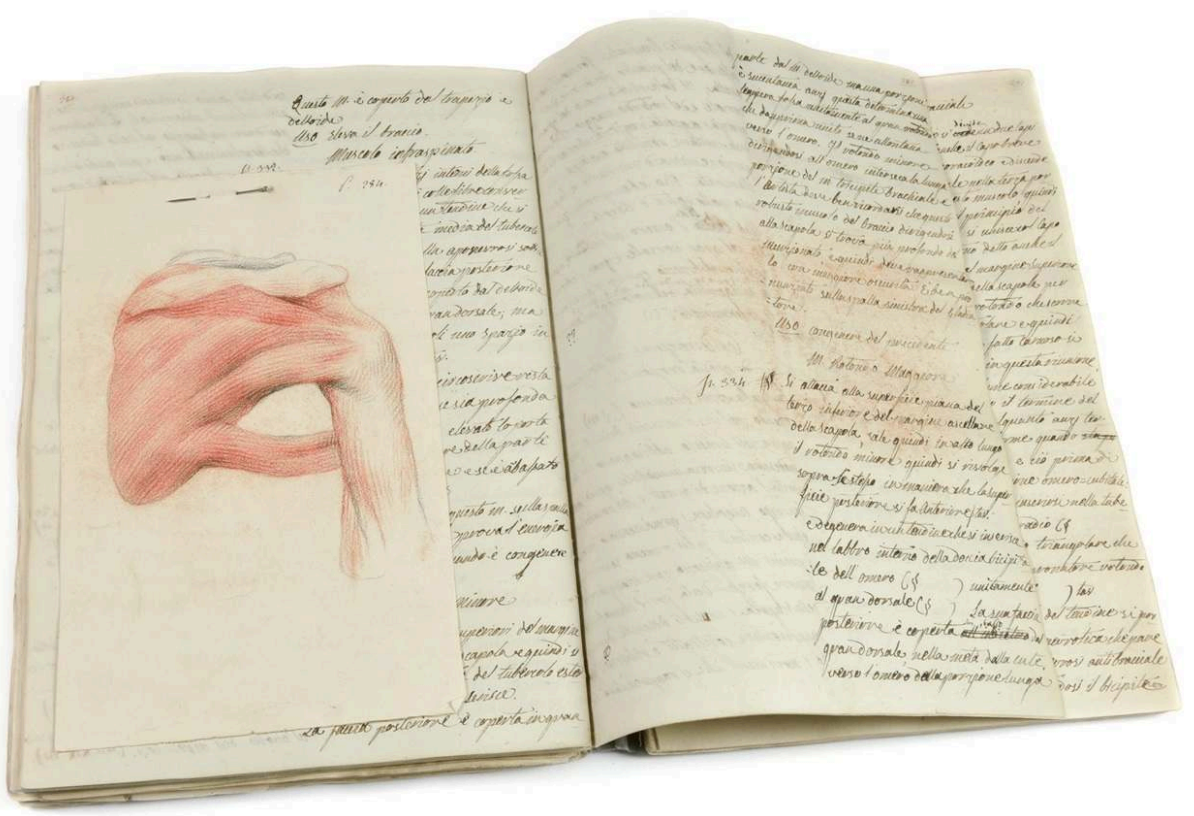

Muscles de l'épaule, Francesco Bertinatti, page 284 de son manuscrit d'anatomie conservé à la bibliothèque de l'Académie.

(c) photo Fabio Amerio (Accademia Albertina di Belle Arti, Turin). 
7 Une « école d'ornements » (« scuola d'ornato ») est confiée au peintre Pelagio Palagi pour améliorer la qualité du travail des artisans employés dans les chantiers des palais royaux $^{14}$. Le marquis Ferdinando Gattinara di Breme est chargé en 1849 de rendre l'enseignement plus libre et plus efficace. Il s'inspire du modèle des ateliers du Moyen Âge, qui offre la possibilité pour les élèves de choisir entre les maîtres, en concurrence. Le corps des anciens enseignants est presque entièrement remplacé par des jeunes de formation internationale, tels Carlo Felice Biscarra, peintre et graveur nommé secrétaire en 1860 , et le sculpteur suisse Vincenzo Vela, tous choisis par di Breme ${ }^{15}$.

8 Francesco Bertinaria a laissé une description détaillée de l'Académie en $1851^{16}$, qui cite les modèles d'architecture et les moulages de détails décoratifs, les dessins et gravures d'œuvres d'art, les collections de livres d'art, les soixante cartons de Gaudenzio Ferrari et autres peintres de la Renaissance donnés par le roi Charles-Albert, les aquarelles de Giuseppe Pietro Bagetti, les modèles d'animaux, les squelettes, les tableaux de la collection familiale donnés par l'archevêque Vincenzo Mossi di Morano ${ }^{17}$.

Dans les années 1860, l'enseignement de la xylographie, liée à l'imagerie populaire et au développement des éditions d'art, prend de l'importance par rapport à la traditionnelle gravure sur cuivre ${ }^{18}$. La décoration sur porcelaine et la petite sculpture en terre cuite comme matières d'enseignement témoignent de l'orientation vers l'artisanat de qualité de la deuxième moitié du siècle ${ }^{19}$. En 1872 , une œuvre collective du maître académicien Odoardo Tabacchi et de ses élèves célèbre le percement du tunnel ferroviaire alpin du Fréjus par un monument qui comprend plusieurs statues titanesques ${ }^{20}$. L'Académie se préoccupe de la sauvegarde des bâtiments et monuments en danger du Moyen Âge avec un premier recensement régional, publié en 1878. Le néo-médiévalisme de la fin du siècle trouve un témoignage éclatant dans la construction, à Turin, au milieu du parc du Valentino, à l'occasion de l'Exposition générale italienne de 1884, d'un concentré de copies d'art et d'architecture piémontaises et valdotaines d'avant la Renaissance, dit "Bourg et château médiéval ${ }^{21}$ ".

10 Ce n'est qu'en 1923, après une longue résistance pour défendre les traditions locales, que les classes préparatoires sont séparées de l'Académie pour donner naissance à un Institut des beaux-arts, conforme à ceux de toute l'Italie ${ }^{22}$. On trouve une description des locaux de l'Académie et des collections dans les années 1920 dans le livre de Luigi Cesare Bollea ${ }^{23}$. En 1941, les peintres Felice Casorati et Enrico Paulucci commencent leur enseignement à l'Académie.

11 Les bombardements de la Deuxième Guerre mondiale provoquent de graves dommages au bâtiment et aux collections, dont celle des moulages, presqu'anéantie. Parmi les œuvres qui ont échappé au désastre, on trouve la copie de la Porte du Paradis réalisée en 1425 par Lorenzo Ghiberti pour le baptistère de Florence [fig. 8]. 
Figure 8

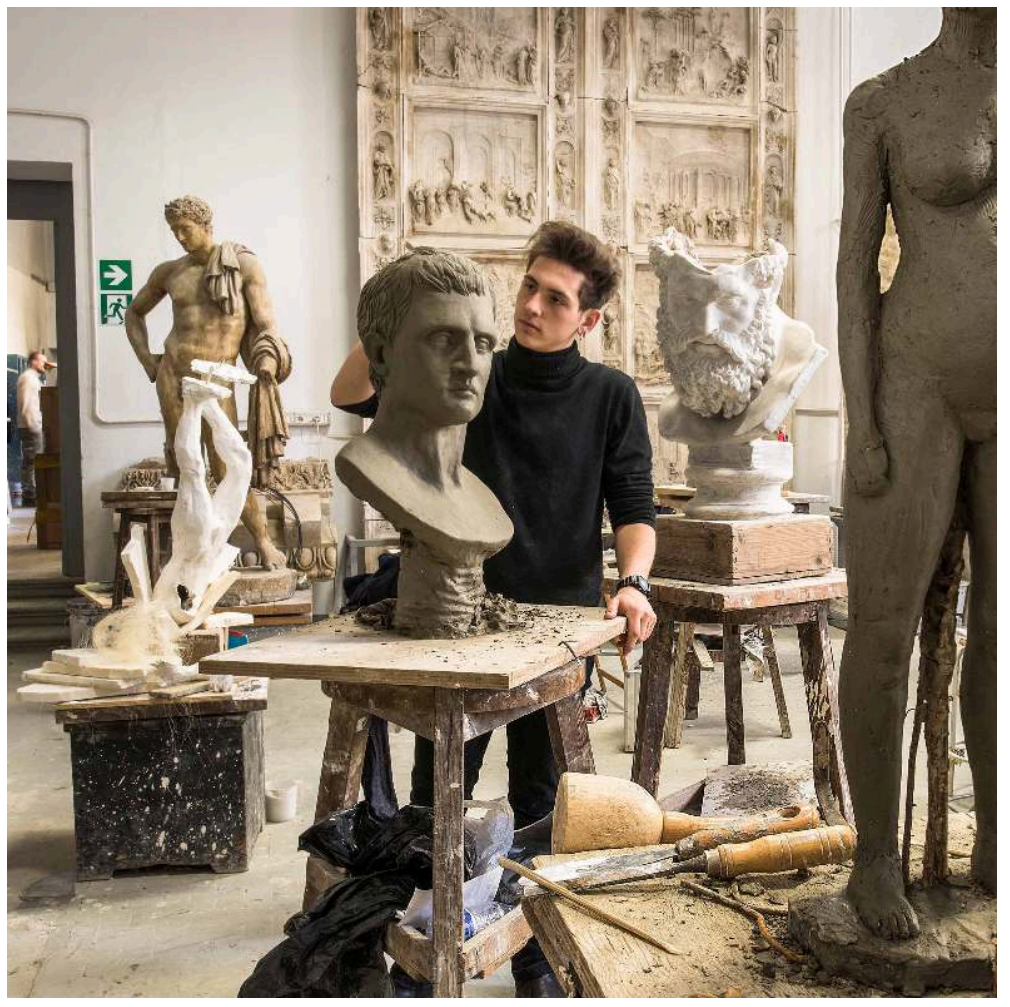

Accademia Albertina, atelier de sculpture. Au mur, moulage de la Porte du paradis sculptée en 1425 par Lorenzo Ghiberti pour le baptistère de Florence.

(c) photo Fabio Amerio (Accademia Albertina di Belle Arti, Turin).

12 Après la Libération, on procède à la remise en état des lieux : le bâtiment est restauré, la galerie de peintures est réorganisée et rouverte. Les travaux de réaménagement technologique de la salle dédiée aux cartons de la Renaissance datent de 2019. La bibliothèque et les archives historiques ont été dotées récemment de catalogues et inventaires revus selon des critères plus scientifiques. L'Académie est aujourd'hui fréquentée par des élèves provenant du monde entier.

\section{Les collections}

13 Les destructions de la guerre se sont ajoutées au manque d'intérêt dont a longtemps fait l'objet la collecte des œuvres des maîtres et élèves de l'Académie. Les instruments actuels de l'enseignement comprennent moulages anciens et modernes (anatomie humaine, statues de l'Antiquité classique, détails décoratifs), peintures, dessins, gravures, photographies, livres, manuscrits scientifiques et artistiques, meubles. Certains documents des archives historiques de l'Académie sont également des témoignages utilisables pour l'enseignement.

\section{La bibliothèque}

14 La bibliothèque ${ }^{24}$ possède un fonds ancien qui remonte aux $\mathrm{XVIII}^{\mathrm{e}}$ et $\mathrm{XIX}^{\mathrm{e}}$ siècles, en majorité des ouvrages d'histoire, de littérature et des recueils d'iconographie, sans oublier la culture classique et religieuse, sources inépuisables de sujets pour l'art. Au cours $\mathrm{du} \mathrm{XIX}^{\mathrm{e}}$ siècle, les ouvrages accumulés sont plus hétérogènes encore car ils 
découlent de nouvelles matières d'enseignement et sont en partie le fruit de donations d'académiciens ou de bienfaiteurs. Ils concernent les sciences de la nature, le paysage, les études d'ingénieur, la médecine, l'anatomie, l'architecture, la décoration, l'histoire ancienne et moderne, la littérature. Des recherches sont en cours pour reconstituer la pédagogie artistique grâce à l'étude de ses instruments ${ }^{25}$. Des volumes portent l'ex-libris de Laurent Pécheux, qui enseignait la peinture et la sculpture en 1778; d'autres viennent de la donation de l'avocat Gerolamo Mattirolo (1847); du fonds parvenu suite à la mort de l'architecte, ingénieur et professeur Alessandro Antonelli (1888); du fonds de l'érudit Piero Alessandro Paravia (fin XIX ${ }^{e}$ siècle). L'achat de plusieurs séries de périodiques est dû au sculpteur et organisateur culturel fasciste Michele Guerrisi pendant la Deuxième Guerre mondiale ${ }^{26}$. Parmi les œuvres acquises à l'époque de Pécheux, on cite un Homère, Les Métamorphoses d'Ovide, une Bible, un Traité de la peinture par Léonard de Vinci avec sa vie par Raphaël Trichet Du Fresne (1786), livre qui porte l'étiquette "À l'usage des élèves de la Royale Académie de peinture et sculpture $^{27}$ ». Le fonds de la bibliothèque de l'Académie sous le directorat de Pécheux ne manque pas d'ouvrages d'histoire orientale, grecque et romaine, avec des détails sur les coutumes religieuses, civiles et militaires (parmi les œuvres inventoriées et étiquetées par l'Académie, celles de Jean Baptiste Louis Crévier et de Michel François Dandré-Bardon), d'ouvrages biographiques dans le style de Giorgio Vasari comme ceux de Filippo Baldinucci (Notizie de' professori del disegno... [1681-1728], Turin, Giuseppe Piacenza, 1768-1820), Bernardo De Dominici (Vite de' pittori, scultori et architetti napoletani, Naples, Francesco \& Cristoforo Ricciardo, 1742-1743), Pellegrino Antonio Orlandi (Abecedario pittorico dei professori più illustri... [1733], Naples, 1775), Anton Raphael Mengs (Opere, publiées par le chevalier José Nicolàs d'Azara, révisées par Carlo Fea et illustrées par le secrétaire de l'Académie milanaise de Brera, Rome, Pagliarini, 1787) ${ }^{28}$.

\section{Le Cabinet des dessins et estampes}

Ce département ${ }^{29}$ a quatre sections : la section ancienne conserve les œuvres du XVI ${ }^{e}$ au XVIII ${ }^{e}$ siècle, la section moderne les œuvres du XIX ${ }^{e}$ siècle, la contemporaine celles du $\mathrm{xx}^{\mathrm{e}}$ siècle (on va y intégrer les épreuves du concours de l'école de gravure de 1950 à 1990), et la section des donations reçues à l'occasion des grandes expositions. Parmi les fonds spéciaux, il y en a qui sont dédiés aux œuvres produites par les membres de la Société des graveurs à l'eau-forte au XIX $\mathrm{e}^{\mathrm{e}}$ siècle, aux cartes et plans, aux fonds Casanova, Boselli et Poli. Le fonds du topographe paysagiste Giuseppe Pietro Bagetti, professeur de l'Académie, témoigne de sa participation aux campagnes napoléoniennes en Italie. On conserve des presses à bras et à levier (pour impression de gravures, lithographies et compositions typographiques) fabriquées à Turin par Bollito et Torchio entre 1860 et 1870 et à Monza par Amos dell'Orto en 1864. Ces presses ont été utilisées pour l'école de gravure depuis la deuxième moitié du XIX siècle jusqu'à nos jours. De 1864 à 1888, la xylographie a été enseignée par le Suisse Giuseppe Salvioni, que l'Académie a envoyé à Paris en 1867 pour étudier les nouveautés techniques en matière de gravure ${ }^{30}$ montrées à l'Exposition universelle.

Les deux sections du fonds photographique, l'ancienne et la moderne, comprennent une série importante de photogravures du début $d u x^{e}$ siècle, à l'usage des élèves ${ }^{31}$. Le patrimoine vidéo, formé par des vidéos d'artiste et des captations de spectacles de théâtre et de ballet, est consultable par les élèves ${ }^{32}$. 


\section{La pinacothèque}

17 Cette collection ${ }^{33}$, qui remonte à 1837, est un des joyaux de l'Académie. Ses douze salles, où peuvent exercer les copistes, sont aussi ouvertes au public [fig. 9]. Elle comprend deux fonds principaux. La donation, en 1828 de 200 tableaux, depuis le $\mathrm{xv}^{\mathrm{e}}$ siècle, par Mgr Vincenzo Mossi di Morano, permet d'exposer, entre autres, des œuvres de Filippo Lippi, Domenico Puligo, Francesco Salviati, Martin van Heemskerck, Defendente Ferrari, Bartolomeo Cavarozzi, Domenico et Anton Maria Piola, Gregorio De Ferrari, Giovanni Battista Carlone. Douze vues de Venise au xvIII siècle sont d'un auteur inconnu.

Figure 9

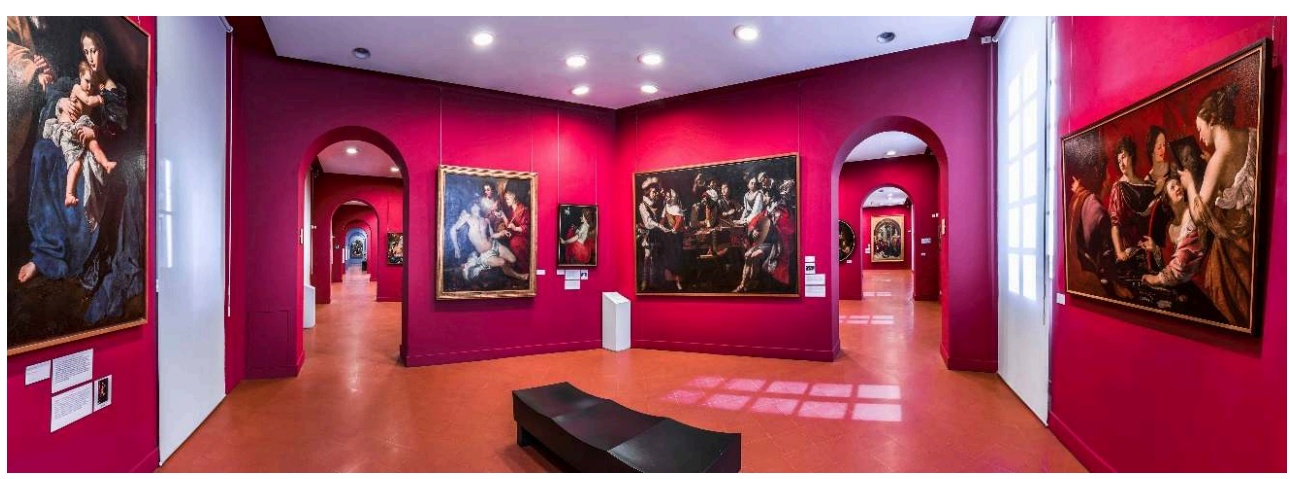

Accademia Albertina, salles de la pinacothèque.

(c) photo Fabio Amerio (Accademia Albertina di Belle Arti, Turin).

Une salle spécialement équipée et éclairée est aujourd'hui consacrée à la donation du roi Charles-Albert, en 1832, de soixante œuvres datées entre 1515 et 1610, issues de l'atelier de Gaudenzio Ferrari et de ses élèves, des cartons et dessins préparatoires de tableaux de la Renaissance [fig. 10]. 
Figure 10

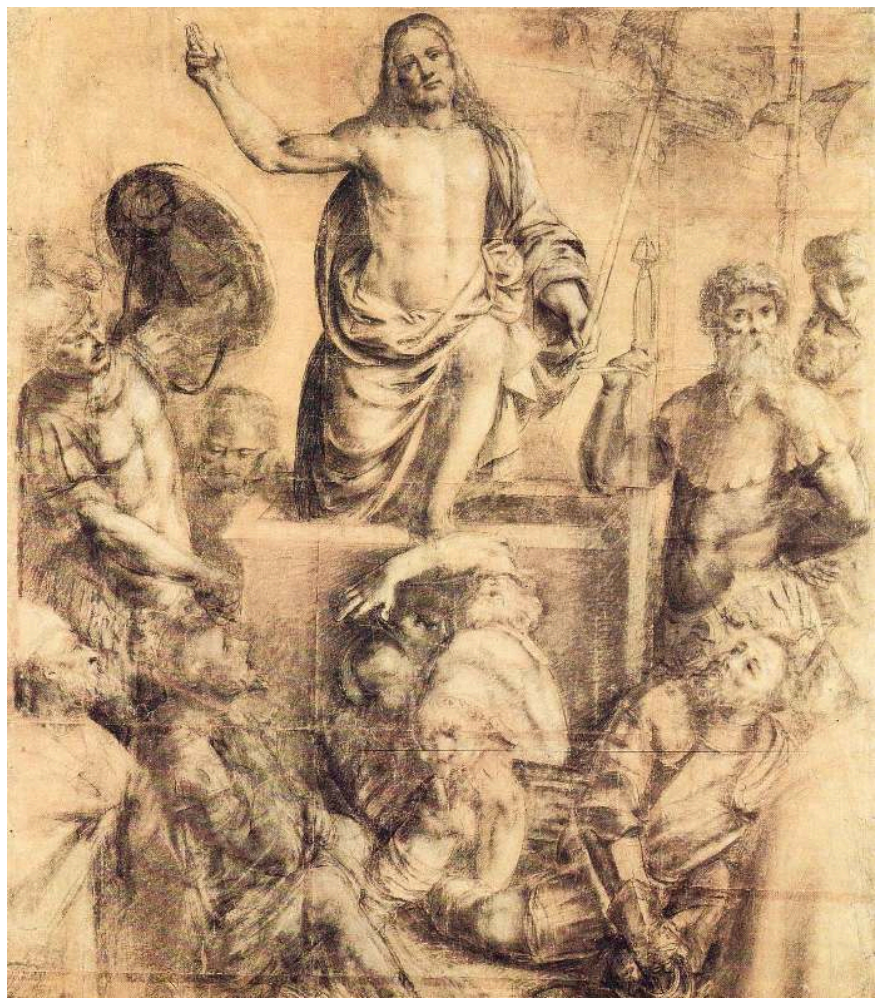

Resurrezione di Cristo e due santi vescovi, Giuseppe Giovenone il Giovane, 1550, carton nº 342 de la collection de l'Académie. Dessin préparatoire du tableau du même sujet conservé à la Galleria Sabauda de Turin.

Reproduction Fabio Amerio (Accademia Albertina di Belle Arti, Turin).

19 D'autres donations, moindres mais assez importantes, sont celles de Giovanni Volpato, enseignant de l'Académie, qui donne en 1835 le tableau $\mathrm{Nu}$ masculin, œuvre de l'atelier de Jacques-Louis David [fig. 11], du peintre Giuseppe Monticoni, qui donne en 1837 sa collection de tableaux du XVII ${ }^{\mathrm{e}}$ siècle, de la veuve du peintre paysagiste Giuseppe Pietro Bagetti (1842) qui donne également le buste en marbre de son mari par Giacomo Spalla, et d'une série d'œuvres des sculpteurs Ignazio et Filippo Collino, appelés à enseigner à l'Académie après la réforme de 1778. 
Figure 11

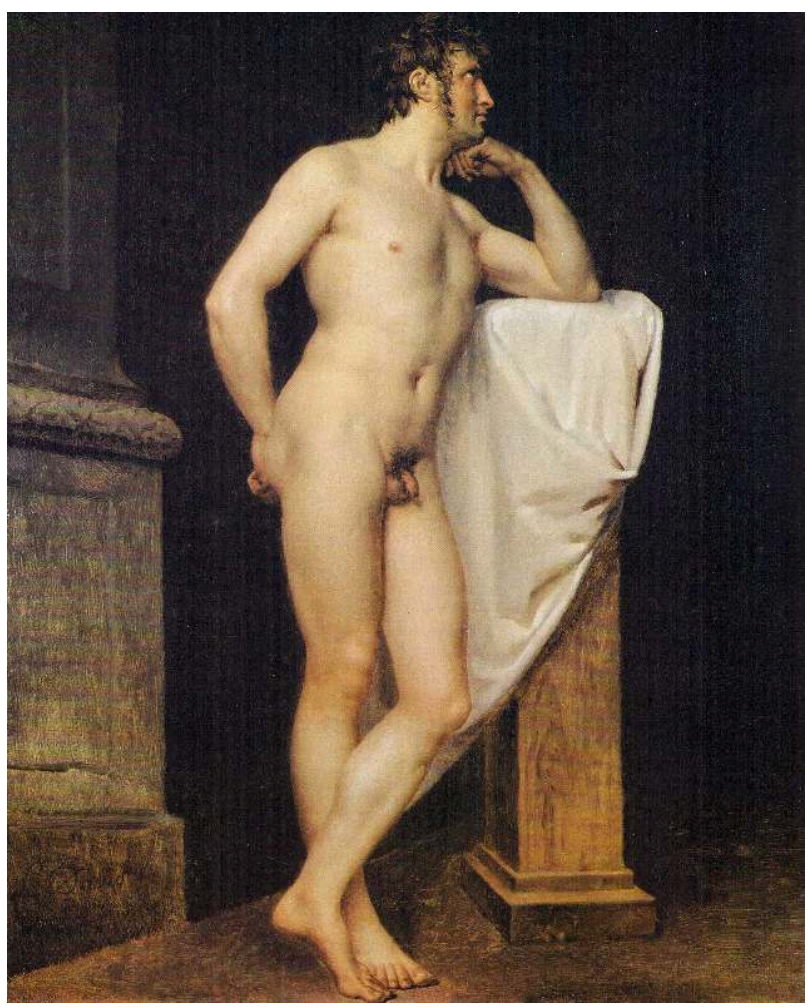

Nu académique, Jacques-Louis David (atelier de), huile sur toile conservée à la Pinacothèque de l'Académie.

Reproduction Fabio Amerio (Accademia Albertina di Belle Arti, Turin).

20 La collection de peintures de Giacomo Grosso (enseignant entre 1906 et 1933) est arrivée par un achat de l'État, en 1939. Elle comprend le tableau La Nue [fig. 12], aujourd'hui en dépôt à la Galerie d'art moderne de Turin. Les fonds du $\mathrm{xx}^{\mathrm{e}}$ siècle concernent de nombreux artistes, parmi lesquels on peut citer Carlo Bonatto Minella, Angelo Beccaria, Bartolomeo Ardy, Ludovico Raymond, Luigi Mainolfi, Luigi Stoisa, Paolo Grassino, Carlo Oreste Strocco. Les œuvres conservées dans les réserves, montées sur châssis roulants, sont visibles sur demande. 
Figure 12

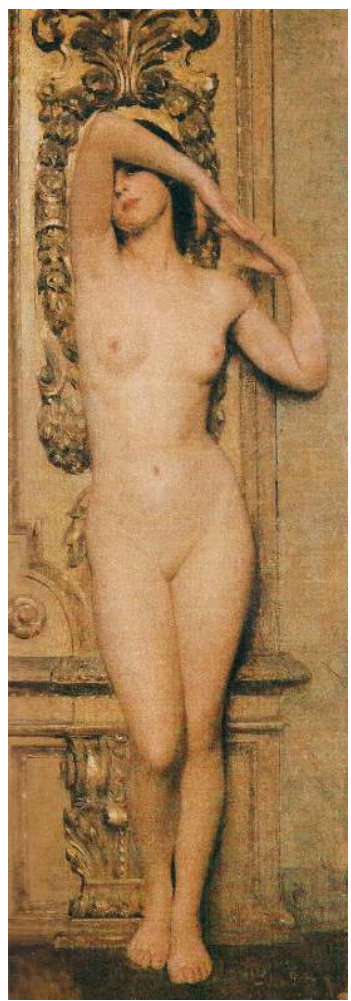

La Nuda, Giacomo Grosso, huile sur toile, dépôt de l'Académie à la Galerie d'art moderne de Turin. Reproduction Fabio Amerio (Accademia Albertina di Belle Arti, Turin).

21 Les bustes de plusieurs personnages importants pour l'histoire de l'Académie sont exposés dans les salons d'honneur. Dans le hall d'entrée se trouve la statue monumentale de l'archevêque Mossi di Morano par le sculpteur Carlo Marocchetti ${ }^{34}$.

\section{La gypsothèque}

Dès la réforme de 1778, l'Académie a accumulé une vaste collection de copies de grandes dimensions de statues grecques et romaines, à commencer par celles commandées à Rome par Laurent Pécheux ${ }^{35}$. Parmi les œuvres qui ont survécu aux bombardements de la dernière guerre, on peut citer la Victoire de Samothrace et le Gladiateur combattant, qui sert à étudier la musculature [fig. 13]. Des acquisitions du $\mathrm{XIX}^{\mathrm{e}}$ siècle concernent, entre autres, des œuvres des sculpteurs Antonio Canova (qui a donné le Torse de Phidias) et Bertel Thorvaldsen, qui a donné la statue de Psyché. Les acquisitions plus nombreuses sont celles dues à Luigi Canina (1843), Giovanni Battista Biscarra (1845), Crescentino Caselli (1894). Il faut noter toutefois que l'utilisation fréquente pour l'enseignement pose des problèmes pour la conservation et la contemplation des collections par le grand public. 
Figure 13

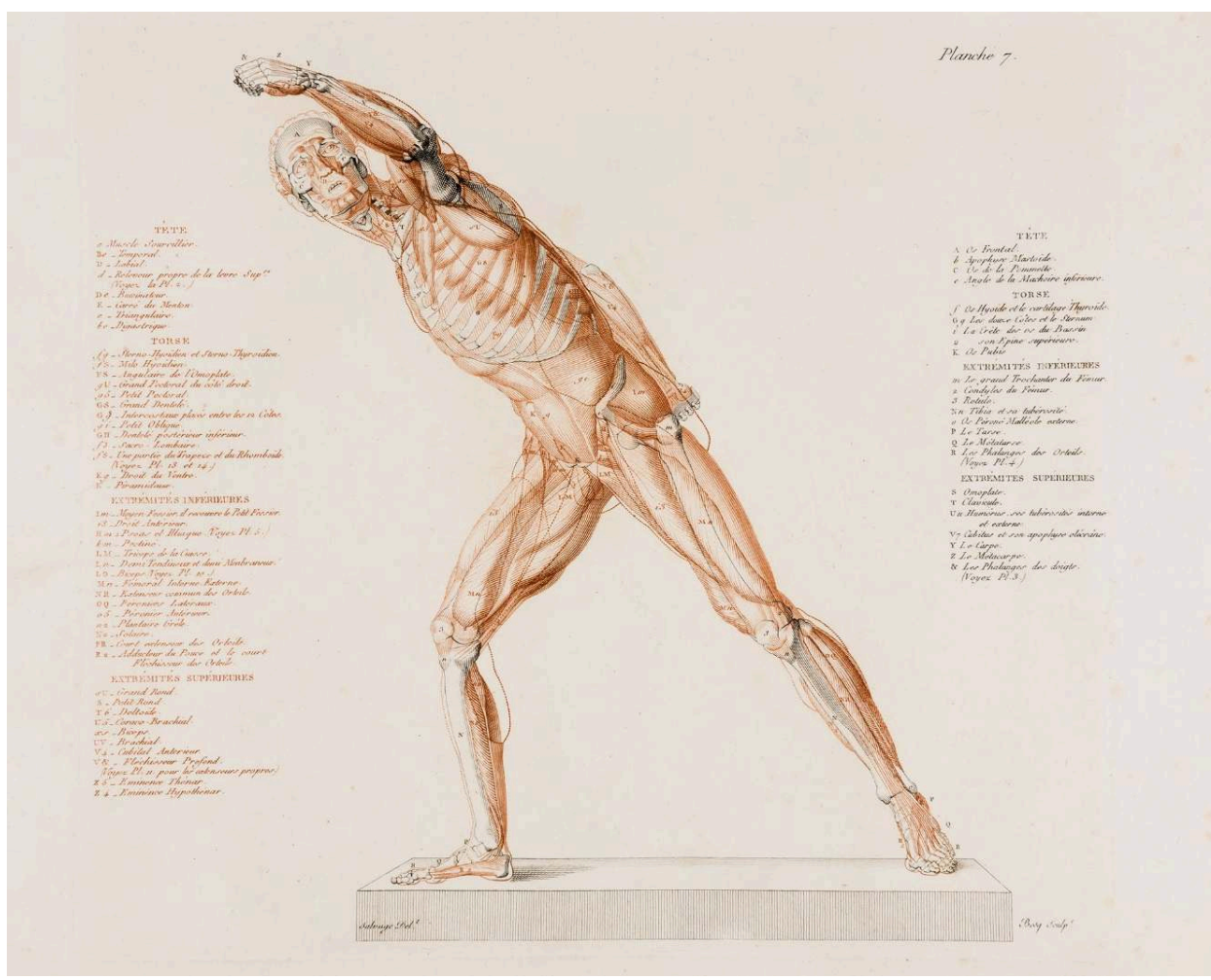

Anatomie du gladiateur combattant, dessin de Jean-Galbert Salvage (médecin militaire de Napoléon) lithographie de Jean Bosq, non datée, planche 7 d'un recueil éditée à Paris, tirage conservé dans la bibliothèque de l'Académie.

Reproduction Fabio Amerio (Accademia Albertina di Belle Arti, Turin).

\section{NOTES}

1. DALMASSO Franca, "L'Accademia Albertina: storia e artisti », in DALMASSO Franca, GAGLIA Pierluigi \& POLI Francesco (dir.), L'Accademia Albertina di Torino, Turin, Arti grafiche, 1982, p. 11.

2. ROBERTO Maria Teresa, «Breve storia dell'Accademia », in CASSESE Giovanna (dir.), Accademie patrimoni di Belle Arti. L'Accademia Albertina di Torino, Rome, Gangemi, 2013, p. 109 et sq.

3. BOLLEA Luigi Cesare, Gli storici dell'Accademia, Turin, Bocca, 1930, p. 45.

4. ROBERTO Maria Teresa, «Breve storia dell'Accademia », p. 109.

5. LALANDE Joseph-Jérôme Lefrançois de, Voyage en Italie, Paris, Veuve Desaint, 1786, p. 245-247.

6. DALMASSO Franca, «L'Accademia Albertina: storia e artisti », p. 24.

7. Ibid., p. 22-28.

8. ROBERTO Maria Teresa, Breve storia dell'Accademia ».

9. POLI Francesco, «La sede dell'Accademia di Belle Arti: l'attuale edificio, precedenti collocazioni, progetti di altre sistemazioni ", in DALMASSO Franca, GAGLIA Pierluigi \& POLI Francesco (dir.), L'Accademia Albertina di Torino, Turin, Arti grafiche, 1982, p. 89 et sq.

10. DALMASSO Franca, «L'Accademia Albertina: storia e artisti », p. 35. 
11. GRASSI Rosella, «Il patrimonio storico-artistico. Breve storia della struttura e degli spazi più rappresentativi ", in CASSESE Giovanna (dir.), Accademie patrimoni di Belle Arti. L'Accademia Albertina di Torino, Rome, Gangemi, 2013, p. 112 et sq.

12. DALMASSO Franca, «L'Accademia Albertina: storia e artisti », p. 34.

13. BERTINATTI Francesco, Elementi di anatomia fisiologica applicata alle Belle Arti figurative, Turin, Pietro Marietti, 1837-1839. Voir MUSIARI Antonio, «Biblioteconomia centripeta. Il nucleo originario degli acquisti di trattati anatomici e fogli a stampa ", in MERLINO Roberta, MUSIARI Antonio, TARTAGLINO Fernanda (dir.), Corpi di carta. Testi e immagini di anatomia nella Biblioteca storica dell'Accademia Albertina, catalogue d'exposition (Turin, Accademia Albertina di Belle Arti di Torino, $1^{\mathrm{er}}$ juillet-8 septembre 2017), Turin, Albertina Press, 2017, p. 13.

14. DALMASSO Franca, «L'Accademia Albertina: storia e artisti », p. 35.

15. DALMASSO Franca, «L'Accademia Albertina: storia e artisti », p. 39.

16. BERTINARIA Francesco, "Una visita alla Reale Accademia Albertina di Belle Arti », Gazzetta piemontese, 21 août 1851.

17. L'archevêque de Turin, proche de la Cour de Savoie, avait hérité de la collection de son frère Tommaso Ottavio Maria, marquis del Torrione, grand voyageur et amateur d'art, mort en 1802.

18. DALMASSO Franca, «L'Accademia Albertina: storia e artisti », p. 46.

19. DALMASSO Franca, «L'Accademia Albertina: storia e artisti », p. 51.

20. DALMASSO Franca, «L'Accademia Albertina: storia e artisti », p. 58.

21. DALMASSO Franca, « L'Accademia Albertina: storia e artisti ».

22. Ibid., p. 70-72.

23. BOLLEA Luigi Cesare, La Regia Accademia Albertina delle belle arti e la Regia casa di Savoia, Turin, Bocca, 1930.

24. POLI Francesco, «La biblioteca: testi didattici, stampe, disegni originali », in DALMASSO Franca, GAGLIA Pierluigi \& POLI Francesco (dir.), L'Accademia Albertina di Torino, Turin, Arti grafiche, 1982, p. 193 et sq.

25. GRASSI Rosella, «La Biblioteca storica », in CASSESE Giovanna (dir.), Accademie patrimoni di Belle Arti. L'Accademia Albertina di Torino, Rome, Gangemi, 2013, p. 115. Rosella Grassi signale que Fabio Cafagna (il a publié en 2017 Il Disegno del corpo. Anatomia artistica all'Accademia Albertina di Torino (1829-1899), Rome, Carocci) étudie l'école d'anatomie et Beatrice Zanelli l'école d'architecture (elle a soutenu en 2016 une thèse d'histoire de l'architecture au Politecnico de Turin, intitulée "Accademia Albertina di belle arti di Torino: vincoli culturali e materiale didattico ad uso degli strudenti della scuola di Alessandro Antonelli (1842-1855) »).

26. GRASSI Rosella, «La Biblioteca storica ». Voir aussi Archives historiques de l'Accademia Albertina (TO 22 [48] et TO 84).

27. GRASSI Rosella, « La Biblioteca storica », p. 116. Il s'agit d'un ensemble de livres identifié par Francesco Poli d'après la liste transcrite par Luigi Cesare Bollea en 1936.

28. GRASSI Rosella, « La Biblioteca storica ».

29. GRASSI Rosella, «Il Gabinetto dei disegni e stampe », in CASSESE Giovanna (dir.), Accademie patrimoni di Belle Arti. L'Accademia Albertina di Torino, Rome, Gangemi, 2013, p. 118-120.

30. SALVIONI Giuseppe, Cenni storici sulla zilografia, ossia incisioni in legno, seguiti da alcune considerazioni intorno alle attuali condizioni di quest'arte in Italia, Turin, Civelli, 1868; cité par FANELLI Franco, "Il patrimonio storico del corso di tecniche dell'incisione e grafica d'arte ", in CASSESE Giovanna (dir.), Accademie patrimoni di Belle Arti. L'Accademia Albertina di Torino, Rome, Gangemi, 2013, p. 122.

31. GRASSI Rosella, « Il Gabinetto dei disegni e stampe », p. 118.

32. AUSONI Alberto, «La videoteca », in CASSESE Giovanna (dir.), Accademie patrimoni di Belle Arti. L'Accademia Albertina di Torino, Rome, Gangemi, 2013, p. 124.

33. PETRUCCI Francesca, "La Pinacoteca Albertina », in CASSESE Giovanna (dir.), Accademie patrimoni di Belle Arti. L'Accademia Albertina di Torino, Rome, Gangemi, 2013, p. 124-126. Voir aussi 
GAGLIA Pierluigi, "La formazione della pinacoteca », in DALMASSO Franca, GAGLIA Pierluigi \& POLI Francesco (dir.), L'Accademia Albertina di Torino, Turin, Arti grafiche, 1982, p. 125 ss.

34. PETRUCCI Francesca, « La Pinacoteca Albertina », p. 126.

35. GRASSI Rosella \& ZANELLI Beatrice, «La Gipsoteca », in CASSESE Giovanna (dir.), Accademie patrimoni di Belle Arti. L'Accademia Albertina di Torino, Rome, Gangemi, 2013, p. 128.

\section{RÉSUMÉS}

Une ancienne corporation de métier reçoit en 1678 le statut d'« Académie des peintres, des sculpteurs et des architectes " de Turin. Les académiciens, hommes et femmes, constituent la première collection d'œuvres d'art destinée à l'enseignement. En 1778, l'Académie est refondée et la direction confiée au peintre français de formation romaine Laurent Pécheux (1729-1821). À l'époque napoléonienne, l'Académie de Turin est rattachée à l'Académie de Paris. En 1833, le roi de Sardaigne Charles-Albert décide l'installation définitive de l'Académie dans son siège actuel. Les inventaires de 1839, 1846 et 1856 témoignent de la croissance de la collection des plâtres, qui atteint 850 œuvres. La bibliothèque s'enrichit de publications et de manuscrits utilisés pour l'enseignement. Après 1849, les élèves peuvent choisir parmi les cours de nouveaux maîtres, en concurrence entre eux.

Une description détaillée de l'Académie en 1851 cite les modèles d'animaux, les squelettes, les modèles d'architecture et les moulages de détails décoratifs, les dessins et gravures d'œuvres d'art, les collections de livres d'art, les aquarelles de Giuseppe Pietro Bagetti, les soixante cartons préparatoires de tableaux de peintres de la Renaissance (placés en 2019 dans une salle spécialement équipée et éclairée), les deux cents tableaux de la collection donnée par l'archevêque Mossi di Morano. Ces deux fonds sont présents dans les douze salles de la pinacothèque actuelle.

Après les bombardements de la dernière guerre, le bâtiment a été restauré, la galerie de peintures réorganisée et rouverte, la gypsothèque reconstituée. La Bibliothèque et les Archives historiques ont été dotées récemment de catalogues et inventaires rénovés. Le Cabinet des dessins et estampes est consultable par les élèves ainsi que le fonds photographique, les vidéos d'artiste et les documentations les captations de spectacles de théâtre et de ballet. L'équilibre entre conservation, utilisation fréquente pour l'enseignement et contemplation des collections pour le grand public reste difficile.

An ancient trade guild was given the charter of Academy of painters, sculptors and architects of Turin in 1678. Academicians, men and women, built the first art collection devoted to teaching. In 1778 the Academy was refounded and a French painter taught by the Roman school, Laurent Pécheux (1729-1821), was appointed director. During the Napoleonic period, the Turin Academy was under the direction of the Paris Academy. In 1833, King Charles Albert of Sardinia chose the definitive location of the Academy, where it still remains today. The inventories of 1839,1846 and 1856 give evidence of the expansion of the plaster models collection, reaching 850 items. The Library has been enriched by publications and manuscripts available for teaching. After 1849, students were allowed to choose among lectures by new competing teachers.

An analytical description of the Academy from 1851 lists animal models, skeletons, architectural models, decoration details casts, drawings and engravings of art works, art books collections, watercolors by Giuseppe Pietro Bagetti, sixty preliminary cartoons for Renaissance pictures 
(placed in a hall especially equipped and illuminated in 2019), two hundred paintings from the Archbishop Mossi di Morano collection. These two groups of art works are shown in the twelve rooms of the present picture-gallery.

After the last war bombings, the palace was restored, the picture-gallery re-organized and reopened, the gypsothèque set up again. Historical Library and Archives were provided with new catalogues and inventories. The drawings and engravings cabinet is accessible for students as well as the photos collections, the artists videos, and the theatre and ballet documentation and capture. Yet, difficulties tend to upset the balance between conservation, frequent use for teaching and enjoyment for general audience.

\section{INDEX}

Mots-clés : Académie de Saint-Luc, Académie de Turin, Académie de Paris, Pécheux (Laurent), néo-médiévalisme, Exposition générale italienne de 1884, cabinet des dessins et estampes, Académie milanaise, Brera, Charles-Félix, Charles-Albert, pinacothèque, gypsothèque Keywords : Academy of Saint-Luc, Academy of Turin, Pécheux (Laurent), Academy of Paris, Charles-Félix, Charles-Albert, Turin Fair 1884, gypsotheque, pinacoteca, Milan Academy, Brera

\section{AUTEUR}

\section{MARCO CARASSI}

Ancien directeur des Archives d'État de Turin

marco.carassi47@gmail.com 